\title{
Metabolic effects of darglitazone, an insulin sensitizer, in NIDDM subjects
}

\author{
R.L. Chaiken ${ }^{1}$, M. Eckert-Norton ${ }^{1}$, R.Pasmantier ${ }^{1}$, G. Boden ${ }^{2}$, I. Ryan ${ }^{2}$, R.A. Gelfand ${ }^{3}$, H. E. Lebovitz ${ }^{1}$ \\ ${ }^{1}$ Department of Medicine, Division of Endocrinology, State University of New York, Health Science Center, Brooklyn, New York, \\ USA \\ ${ }^{2}$ Department of Medicine, Division of Endocrinology and Metabolism, Temple University School of Medicine, Philadelphia, \\ Pennsylvania, USA \\ ${ }^{3}$ Experimental Medicine, Central Research Division, Pfizer Inc., Groton, Connecticut, USA
}

Summary Insulin resistance is a significant pathogenetic factor in the development of non-insulin-dependent diabetes mellitus (NIDDM). A new class of drugs, the thiazolidinediones, have been shown to lower blood glucose levels without stimulating insulin secretion. We report the metabolic effect of the thiazolidinedione, darglitazone, in obese NIDDM subjects. Nineteen subjects were enrolled in a doubleblind placebo-controlled study in which $25 \mathrm{mg}$ of darglitazone was given once a day for 14 days. Nine subjects received the active drug and ten subjects received placebo. Darglitazone-treated subjects showed; 1) a decrease in 24-h plasma glucose area under the curve from $292.8 \pm 31.2$ to $235.2 \pm$ $21.6 \mathrm{mmol} \cdot \mathrm{h}^{-1} \cdot \mathrm{l}^{-1} p=0.002 ; 2$ ) a decrease in $24-\mathrm{h}$ serum insulin area under the curve from 1027.2 \pm
254.4 to $\left.765.6 \pm 170.4 \mu \mathrm{U} \cdot \mathbf{h}^{-1} \cdot \mathbf{1}^{-1} p=0.045 ; 3\right) \mathrm{a}$ decrease in 24 -h non-esterified fatty acid area under the curve from $1900 \pm 236$ to $947 \pm 63 \mathrm{~g} \cdot \mathrm{h}^{-1} \cdot \mathrm{l}^{-1}$ $p=0.002 ; 4$ ) a decrease in mean 24 -h serum triglyceride by $25.9 \pm 6.2 \%$ as compared to $-3.9 \pm 4.8 \%$ for the placebo-treated group, $p=0.012$. Placebo-treated subjects showed no change in their metabolic parameters after treatment. Thus, darglitazone is effective in increasing insulin effectiveness in obese NIDDM subjects. The potential for this and similar drugs to treat or prevent NIDDM as well as the insulin-resistance syndrome needs to be explored. [Diabetologia (1995) 38: 1307-1312]

Key words Non-insulin-dependent diabetes mellitus, darglitazone, insulin sensitizer.
Insulin resistance is a significant contributing factor for many patients in the pathogenesis of non-insulindependent diabetes mellitus (NIDDM) [1-5]. It may be the consequence of a primary genetic abnormality of enzymes regulating muscle glucose metabolism or be secondary to other metabolic abnormalities such as obesity [2, 6-8]. Insulin resistance and/or the associated hyperinsulinaemia are postulated to play a role in the development of hypertension and accelerated atherosclerosis [2, 8-12].

Received: 14 October 1994 and in revised form: 4 May 1995

Corresponding author: Dr. R.L.Chaiken, Box 123, SUNYHSC Brooklyn, 450 Clarkson Avenue, Brooklyn, N. Y. 11203 USA

Abbreviations: NIDDM, Non-insulin-dependent diabetes mellitus; NEFA, non-esterified fatty acids; IVGTT, intravenous glucose tolerance test; AUC, area under the curve.
In the 1970s, a new class of anti-diabetic drugs (thiazolidinediones) was synthesized. The prototype, ciglitazone, was shown to lower blood glucose levels without stimulating insulin secretion [13-17]. Several related drugs have been evaluated in animal models of diabetes and have totally or partially corrected existing hyperglycaemia and hyperinsulinaemia [1820]. Reductions in serum triglyceride and non-esterified fatty acid (NEFA) levels have accompanied the reduction in serum insulin and glucose. Thus, these drugs are postulated to improve glucose metabolism in experimental diabetes by increasing insulin action in insulin-sensitive tissues.

If such agents were to have similar effects in humans, the potential therapeutic use could include prevention as well as treatment of NIDDM and perhaps amelioration of the insulin resistance syndrome. Clinical studies of the effect of one of these agents, troglitazone, have been reported [21-23]. Some improvement 
in insulin sensitivity and glycaemic control was shown in NIDDM subjects and non-diabetic obese subjects with either normal or impaired glucose tolerance.

This report describes the metabolic effects of another thiazolidinedione derivative, darglitazone, in a double-blind placebo-controlled study evaluating the effect of $25 \mathrm{mg}$ taken once a day for 14 days in 19 obese NIDDM subjects.

\section{Subjects and methods}

Nineteen subjects with NIDDM as defined by the Diabetes Data Group [24] participated in the study. Subjects had a duration of NIDDM of 6 months or greater, and had been treated with sulphonylurea agents and/or diet. All subjects were obese (BMI $27-40 \mathrm{~kg} / \mathrm{m}^{2}$ ), and had some insulin secretory reserve as assessed by $\mathrm{C}$-peptide response to glucagon. A minimal plasma C-peptide level greater than $0.8 \mathrm{nmol} / 1$ measured $6 \mathrm{~min}$ after administration of $1 \mathrm{mg}$ of intravenous glucagon was required for acceptance into the study. Subjects were otherwise healthy and had no major complications of NIDDM. No subject was taking medication that would interfere with glucose control. Informed consent was obtained from all subjects at the start of the study. Oral sulphonylurea therapy was discontinued and subjects were instructed in a weight-maintaining diabetic diet for 3 weeks prior to the first hospital visit.

Study protocol. Subjects were admitted to the Clinical Research Centers at State University of New York - Health Science Center at Brooklyn, New York, USA and Temple University School of Medicine, Philadelphia, Pennsylvania, USA for the initial baseline studies. On day 1,24 -h profiles were performed for plasma glucose, serum insulin, serum C-peptide, serum triglycerides and plasma NEFA. For plasma glucose and serum insulin, 13 samples were taken throughout the day between 07.00 and 03.00 hours the following day. Twelve samples were obtained for serum C-peptide, and seven samples were obtained for both serum triglycerides and plasma NEFA. The breakfast meal was Sustacal liquid formula delivering $25 \%$ of the day's total kcal. Lunch and dinner delivered 35 and $40 \%$, respectively of the kcal for the day. On day 2 , subjects underwent an intravenous glucose tolerance test (IVGTT) (25 g dextrose delivered over $2 \mathrm{~min}$ ) [25]. During the IVGTT 15 samples for glucose and insulin determinations were obtained. Subjects were then randomized in a double-blind manner into groups receiving either $25 \mathrm{mg}$ darglitazone or placebo and treated each morning for 2 weeks including the subsequent test days. They were readmitted to the Clinical Research Centers at the end of the 2 weeks for a repeat of the baseline studies.

Analytical methods. Plasma glucose was measured by a glucose oxidase method using a Beckman glucose analyzer (Brea, Calif., USA). Serum insulin and C-peptide were measured by double antibody methods for radioimmunoassay for each peptide using the Incstar RIA kits (Stillwater, Minn., USA). NEFA were measured on EDTA-plasma using an in vitro enzymatic colorimetric method [26]. Serum triglyceride was measured enzymatically using the Cobas Mira Instrument (Montclair, N.J., USA).

\section{Statistical analysis}

Data were analysed using the paired and two-sample Student's $t$-test. All data are presented as mean \pm SEM.
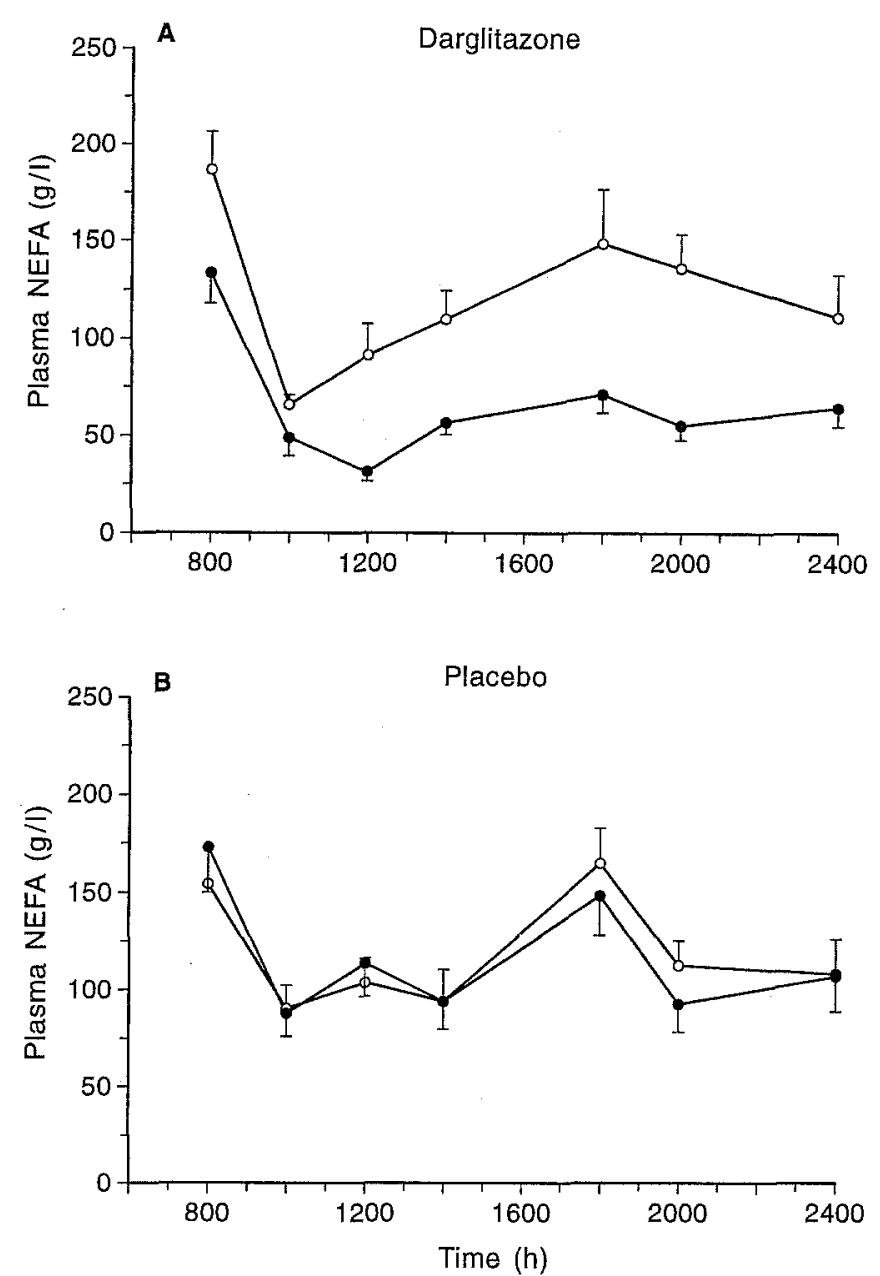

Fig. 1 (A, B). 24-h plasma NEFA profiles (A) for darglitazonetreated, (B) for placebo-treated patients. O Baseline 24 -h profile; post-treatment profiles

\section{Results}

Nine subjects received the active drug, darglitazone, and ten subjects received placebo. Both groups had the same degree of obesity (BMI $31.2 \pm 2.8$ vs $30.3 \pm 1.0 \mathrm{~kg} / \mathrm{m}^{2}$, active drug vs placebo, respectively). The group which received darglitazone was significantly younger than the placebo-treated group $(52.0 \pm 2.8$ vs $59.5 \pm 2.2$ years, $p=0.045)$ though duration of NIDDM was not statistically different. Baseline 24-h area under the curve (AUC) of plasma glucose and NEFA and serum insulin, C-peptide, and triglycerides did not differ between the darglitazone and placebo-treated groups.

Plasma NEFA. The baseline 24-h plasma NEFA profiles in the darglitazone and placebo-treated groups were virtually identical (Fig. 1). The 24-h plasma AUCs were $1900 \pm 95$ vs $1842 \pm 184 \mathrm{~g}$. $\mathrm{h}^{-1} \cdot \mathrm{1}^{-1}$ respectively. Placebo treatment did not change the profile (AUC $1759 \pm 220 \mathrm{~g} \cdot \mathrm{h}^{-1} \cdot \mathrm{l}^{-1}$, $p=0.530$, Fig. $1, \mathrm{~B})$. However, in the darglitazone- 

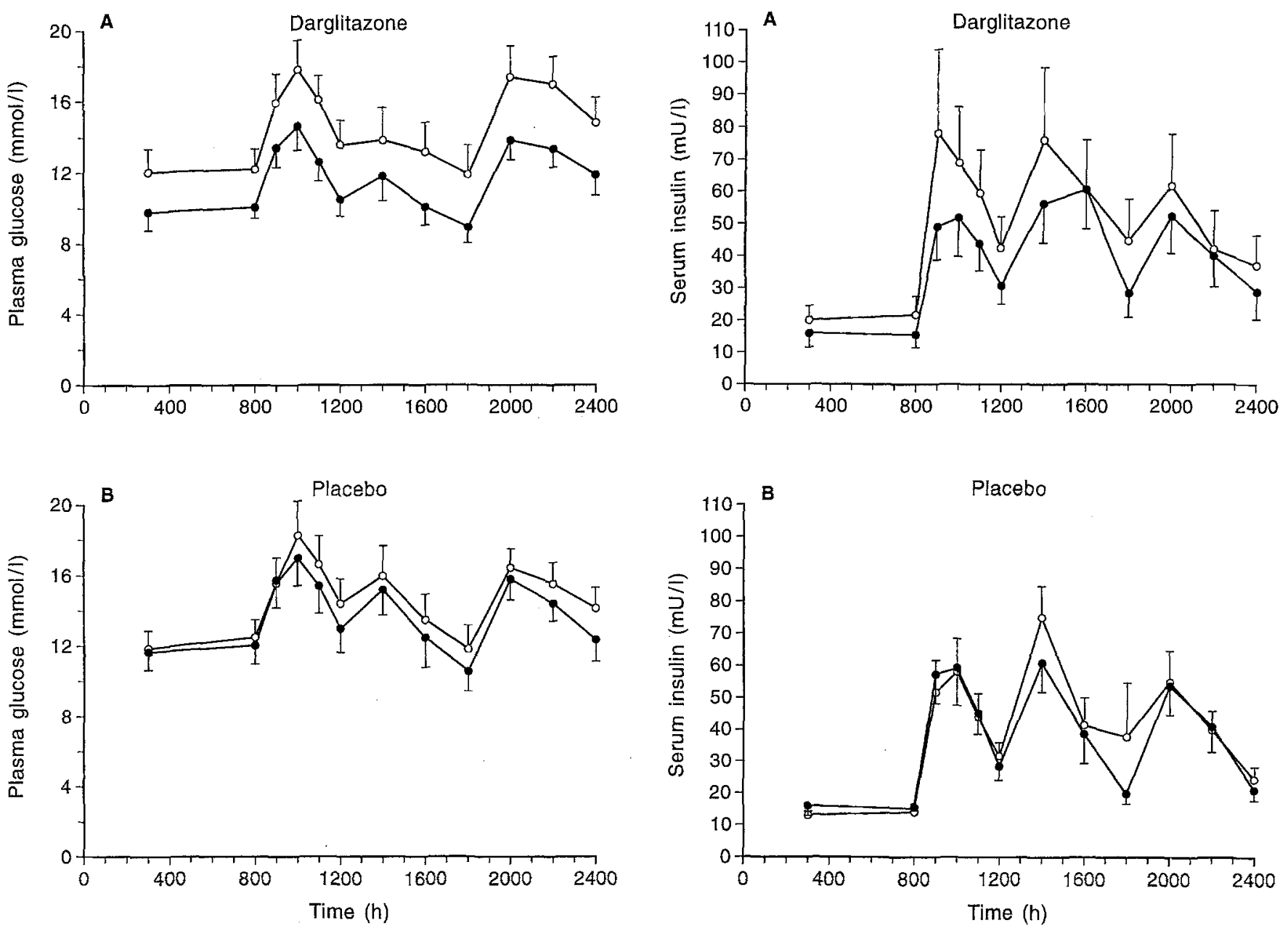

Fig. 2(A,B). 24-h plasma glucose profiles (A) for darglitazone-treated, (B) for placebo-treated patients. O Baseline 24h profile; - post-treatment profiles

treated group, the 24-h plasma NEFA levels were significantly reduced (AUC $947 \pm 63 \mathrm{~g} \cdot \mathrm{h}^{-1} \cdot \mathrm{l}^{-1}$, $p=0.002$, Fig. 1, A). This was different from placebo treatment at $p=0.004$, Figure 1 . Meal-related plasma NEFA levels decreased most in the darglitazone-treated group, Figure 1. The percent decrease in 24-h plasma NEFA AUC from baseline in the darglitazone-treated group as compared to the placebo-treated group was $-45.7 \pm 5.5$ vs $-4.5 \pm 7.2 \%$, $p=0.0003$.

Glucose metabolism. Mean fasting plasma glucose level decreased from $11.9 \pm 0.8$ to $10.1 \pm 0.7 \mathrm{mmol} / 1$ after 2 weeks of darglitazone-treatment while in the placebo-treated group mean fasting plasma glucose did not change $(12.2 \pm 0.9-11.9 \pm 1.1 \mathrm{mmol} / \mathrm{l})$ after 2 weeks. The 24-h plasma glucose AUC in the darglitazone-treated patients decreased from $292.8 \pm 31.2$ to $235.2 \pm 21.6 \mathrm{mmol} \cdot \mathrm{h}^{-1} \cdot \mathrm{l}^{-1}, p=0.002$ and was different from the placebo-treated group, $p=0.02$. The AUC did not change for the placebo-treated group, $283.2 \pm 26.4$ to $266.4 \pm 26.4 \mathrm{mmol} \cdot \mathrm{h}^{-1} \cdot \mathrm{l}^{-1}, p=0.125$,

Fig.3 (A, B). 24-h serum insulin profiles (A) for darglitazonetreated, (B) for placebo-treated patients. O Baseline 24-h profile; post-treatment profiles

Figure 2. The percent change in mean 24-h serum glucose was $-19.3 \pm 3.8 \%$ for the darglitazone-treated group and $-5.6 \pm 3.4 \%$ for the placebo-treated group, $p=0.008$.

Insulin secretion. The 24-h serum insulin AUC decreased $17.7 \pm 6.1 \%$ in the darglitazone-treated group from $1027.2 \pm 254.4$ to $765.6 \pm 170.4 \mathrm{mU}$. $\mathrm{h}^{-1} \cdot \mathrm{l}^{-1}, p=0.045$. It did not change in the placebotreated group, $753.6 \pm 84.0$ to $727.2 \pm 96.0 \mathrm{mU} \cdot \mathrm{h}^{-1}$. $1^{-1},(-3.2 \pm 7.4 \%), p=0.67$, Figure 3 . The change in 24-h insulin AUC between the darglitazone and placebo-treated groups failed to achieve statistical significance $(p=0.07)$.

C-peptide secretion. The change in 24-h serum C-peptide AUC was not significantly different in either the darglitazone-treated or the placebo-treated groups $\left(46.56 \pm 7.20\right.$ to $39.12 \pm 4.32 \mathrm{ng} \cdot \mathrm{h}^{-1} \cdot \mathrm{ml}^{-1},-11.0 \pm$ $4.7 \%, p=0.07$ and $54.00 \pm 6.48$ to $57.60 \pm 6.24 \mathrm{ng}$. $\mathrm{h}^{-1} \cdot \mathrm{ml}^{-1}, 7.4 \pm 3.2 \%, p=0.08$, respectively), Figure 4 . However, the difference in the change in C- 

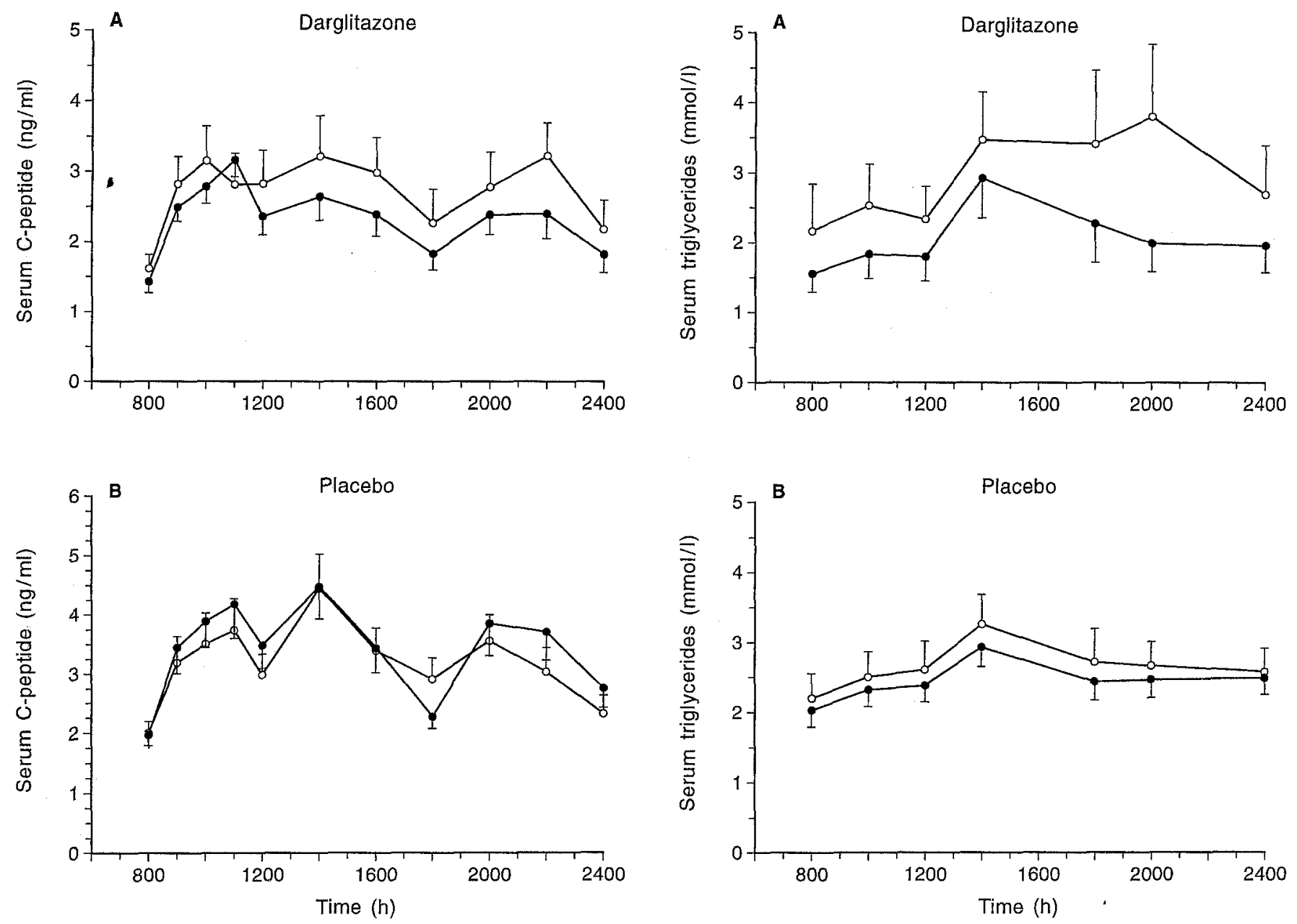

Fig.4(A,B). 24-h serum C-peptide profiles (A) for darglitazone-treated (B) for placebo-treated patients. O Baseline 24-h profile; $\bullet$ post-treatment profiles

Fig.5(A, B). 24-h serum triglyceride profiles (A) for darglitazone treated and (B) for placebo-treated patients. $O$ Baseline 24-h profile; $\bullet$ post-treatment profiles

peptide between the two groups was significant, $p=0.018$.

Triglyceride metabolism. The 24-h serum triglyceride profiles are shown in Figure 5. Placebo treatment had no effect on either fasting serum triglyceride or the serum triglyceride response to meals (Fig. 5B). Although darglitazone treatment resulted in a decrease in serum triglycerides at all times during the 24-h profile, with the most dramatic decreases being noted in the evening (Fig. $5 \mathrm{~A}$ ), it failed to achieve statistical significance $(p=0.07)$. The percent change, however, in mean $24-\mathrm{h}$ serum triglyceride was statistically significant for the darglitazone-treated group, $25.9 \pm 6.2 \%$, vs $-3.9 \pm 4.8 \%$ for the placebo-treated group, $p=0.012$.

IVGTT response. The rate of glucose disappearance $\left(\mathrm{K}_{\mathrm{g}}\right)$ in response to an i. v. bolus of glucose was no different at baseline for either the darglitazone- or placebo-treated groups (Table 1 ). $\mathbf{K}_{\mathrm{g}}$ did not change with either darglitazone or placebo treatment. The

mean fasting serum insulin level at baseline was similar between the darglitazone and placebo-treated groups, $17.8 \pm 6.6$ vs $15.4 \pm 2.0 \mu \mathrm{U} / \mathrm{ml}$, respectively. Neither treatment changed the fasting serum insulin level, or the sum of the 1-, 5-, and 10-min serum insulin levels in response to i.v. glucose (Table 1). Thus, as anticipated, the insulin secretory response to i.v. glucose at baseline was very low. Darglitazone had no effect on either insulin secretion or glucose disposal in response to i. v. glucose.

\section{Discussion}

In this double-blind placebo-controlled trial in obese NIDDM subjects our data show that 14 days of treatment with the thiazolidinedione derivative, darglitazone, is effective in 1) decreasing mean fasting plasma glucose levels; 2) decreasing the 24-h AUC for plasma glucose, serum insulin and C-peptide; 3) decreasing the 24-h plasma AUC for NEFA, and 4) decreasing mean serum triglyceride levels. 
Table 1. $\mathrm{K}_{\mathrm{G}}$ and insulin responses to intravenous glucose in darglitazone- and placebo-treated subjects

\begin{tabular}{|c|c|c|c|c|}
\hline & \multicolumn{2}{|c|}{ Darglitazone $(n=8)$} & \multicolumn{2}{|c|}{ Placebo $(n=10)$} \\
\hline & Baseline & $\begin{array}{l}\text { After } \\
\text { treatment }\end{array}$ & Baseline & $\begin{array}{l}\text { After } \\
\text { treatment }\end{array}$ \\
\hline $\mathrm{K}_{\mathrm{G}}$ & $\begin{array}{l}-0.313 \\
\pm 0.081\end{array}$ & $\begin{array}{l}-0.348 \\
\pm 0.045\end{array}$ & $\begin{array}{l}-0.441 \\
\pm 0.044\end{array}$ & $\begin{array}{l}-0.409 \\
\pm 0.034\end{array}$ \\
\hline $\begin{array}{l}\text { Sum of 1-, 5-, } \\
10-\text { min insulin } \\
(\mathrm{mU} / \mathrm{l})\end{array}$ & $\begin{array}{r}52.2 \\
+21.0\end{array}$ & $\begin{array}{r}47.3 \\
\pm 12.2\end{array}$ & $\begin{array}{r}37.1 \\
\pm 4.5\end{array}$ & $\begin{array}{r}40.0 \\
\pm 3.6\end{array}$ \\
\hline
\end{tabular}

Data are mean \pm SEM

Thiazolidinediones are a class of drugs which have been shown to lower blood glucose levels by decreasing insulin resistance. In studies in $\mathrm{ob} / \mathrm{ob}$ and $\mathrm{db} / \mathrm{db}$ mice, diabetic Chinese hamsters, and normal and streptozotocin-diabetic rats, ciglitazone was found to be effective in lowering blood glucose in the hyperglycaemic hyperinsulinaemic models, but had little effect in the insulin-deficient diabetic models [1317]. The decrease in blood glucose levels was coupled with a corresponding decrease in plasma triglyceride and NEFA levels. Urinary C-peptide levels decreased significantly, though the decrease in insulin levels was not significant.

Ciglitazone increased 2-deoxy-D-glucose uptake in skeletal muscle from ob/ob mice while it increased basal and insulin-stimulated lipogenesis in adipocytes from the treated animals, thus suggesting a mechanism for the decrease in NEFA.

Englitazone, which is the most similar drug to darglitazone, lowered plasma glucose and insulin levels in ob/ob mice [20]. In vitro, using 3T3-L1 adipocytes, englitazone had insulinomimetic properties as shown by its ability to stimulate 2-deoxy-D-glucose uptake in the absence of insulin [18-19]. In further studies englitazone increased glucose transporter mRNA levels in parallel with increased amounts of glucose transporter protein [19]. This suggests that englitazone induces de novo synthesis of glucose transporters. Thus, the drug has both insulinomimetic and insulin sensitizing actions.

There have been several reports on the use of troglitazone in humans with NIDDM and either normal or impaired glucose tolerance [21-23]. Suter et al. [21] reported on the metabolic effects of troglitazone in subjects with NIDDM. Eleven subjects were treated for 6-12 weeks; eight subjects responded favourably with a decrease in oral glucose tolerance test AUC and a fall in post prandial glucose, insulin, NEFA, and glucagon levels. Nolan et al. [23] treated obese non-diabetic subjects with normal or impaired glucose tolerance with troglitazone or placebo. The troglitazone-treated subjects demonstrated an increase in insulin sensitivity as determined by the hyperinsulinaemic euglycaemic clamp technique and the insulin sensitivity index using the
IVGTT, and an improvement in glucose tolerance with decreasing insulin levels in response to meal tolerance tests.

In our darglitazone-treated subjects, 24 -h serum glucose profiles decreased as did 24 -h serum insulin and C-peptide levels. This suggests that the improvement in glycaemia results from improvement in insulin action though this hypothesis needs to be directly tested. In addition, darglitazone does not have an independent insulinomimetic effect on glucose disposal as the $\mathrm{K}_{\mathrm{G}}$ value and the insulin response to intravenous glucose did not change with darglitazone treatment. The failure of darglitazone to increase glucose uptake during the IVGTT demonstrates the need for circulating insulin for darglitazone's glucose-lowering effect.

The decrease in 24-h NEFA profile indicates that darglitazone enhances lipogenesis in the adipose tissue and/or decreases lipolysis either by a direct effect or through improved insulin sensitivity. Since the serum concentration of insulin which affects lipolysis is significantly lower than that which stimulates glucose transport, changes in insulin sensitivity in the presence of impaired insulin secretion will have a more profound effect on lipid metabolism as compared to glucose metabolism. As NEFA are precursors for triglyceride synthesis and postprandial serum triglyceride levels are decreased, this drug may have a significant anti-atherogenic potential. Reports have suggested a relationship between serum triglyceride levels with an increased risk for coronary heart disease [27].

Our data in this placebo-controlled double-blind study indicate that darglitazone increases insulin effectiveness in NIDDM patients. The potential for this and similar drugs to prevent or treat NIDDM needs to be further explored. Their benefits may include partial amelioration of the insulin resistance syndrome and some anti-atherogenic actions.

Acknowledgements. We thank Ms. E. Lewis, RD for expert nutrition counselling of the study subjects. We acknowledge the assistance of the excellent nurses and staff of the Clinical Research Centers at University Hospital at Brooklyn and at Temple University School of Medicine. We thank Dr. E. Chan for assistance with data analysis.

These studies were supported by grants from the Clinical Research Center branch of the Division of Research Resources (RR-00318 and 00349) of the National Institutes of Health, Pfizer Inc., USPHS R01 07988, and the ADA Clinical Research Grant.

\section{References}

1. DeFronzo RA (1988) The triumvirate: beta cell, muscle, liver. A collusion responsible for NIDDM. Diabetes 37: 667-687

2. Reaven GM (1988) The role of insulin resistance in human disease. Diabetes 37: 1595-1607 
3. Banerji MA, Lebovitz HE (1989) Insulin-sensitive and insulin resistant variants in NIDDM. Diabetes 38: 784-792

4. Chaiken RL, Banerji MA, Pasmantier R et al. (1991) Patterns of glucose and lipid abnormalities in black NIDDM subjects. Diabetes Care 14: 1036-1042

5. Chaiken RL, Banerji MA, Huey H, Lebovitz HE (1993) Do blacks with NIDDM have an insulin-resistance syndrome? Diabetes 42: 444-449

6. Kolterman OG, Insel J, Saekow M et al. (1980) Mechanisms of insulin resistance in human obesity. J Clin. Invest 65: 1272-1284

7. Banerji MA, Lebovitz HE (1992) Insulin action in black Americans with NIDDM. Diabetes Care 10: 1295-1302

8. Ferrannini E, Buzzigoli G, Bonadonna R, Giorico MA et al. (1987) Insulin resistance in essential hypertension. $\mathrm{N}$ Engl J Med 317: 350-357

9. Zavaroni I, Bonora E, Pagliara M et al. (1989) Risk factors for coronary artery disease in healthy persons with hyperinsulinemia and normal glucose tolerance. $\mathrm{N}$ Engl J Med 320: 702-706

10. Banerji MA, Lebovitz HE (1991) Coronary heart disease risk factor profiles in black NIDDM patients: paradoxical patterns. Am J Med 91: 51-58

11. Saad MF, Lillioja S, Nyomba BL et al. (1991) Racial differences in the relation between blood pressure and insulin resistance. N Engl J Med 324: 733-739

12. Stout RW (1990) Insulin and atheroma-20-yr prospective. Diabetes Care 13: 631-654

13. Chang AY, Wyse BM, Gilchrist BJ et al. (1983) Ciglitazone, a new hypoglycemic agent $\mathrm{I}$ : Studies in ob/ob and $\mathrm{db} / \mathrm{db}$ mice, diabetic Chinese hamsters, and normal and streptozotocin-diabetic rats. Diabetes 32: 830-838

14. Fujita T, Sugiyama Y, Sohda Tet al. (1983) Reduction of insulin resistance in obese and/or diabetic animals by 5-[4-(1methylcyclohexylemthoxy)benzyl]-thiazolodine-2,4-dione (ADD-3878, U-63,287, Ciglitazone), a new antidiabetic agent. Diabetes 32: 804-810

15. Colca JR, Wyse BM, Sawada G et al. (1988) Ciglitazone, a hypoglycemic agent: early effects on the pancreatic islets of ob/ob mice. Metabolism 37: 276-280
16. Chang AY, Wyse BM, Gilchrist BJ (1983) Ciglitazone, a new hypoglycemic agent II: effect on glucose and lipid metabolism and insulin binding in the adipose tissue of C57BL/6J-ob/ob and $-+/$ ? mice. Diabetes 32: 839-845

17. Shargill NS, Tatoyan A, Fukushima M et al. (1986) Effect of ciglitazone on glucose uptake and insulin sensitivity in skeletal muscle of the obese (ob/ob) mouse: distinct insulin and glucocorticoid effects. Metabolism 35: 64-70

18. Kreutter DK, Andrews KM, Gibbs EM et al. (1990) Insulinlike activity of new antidiabetic agent CP 68722 in 3T3L1 adipocytes. Diabetes 39: 1414-1419

19. Gibbs EM, Genereux PE, Kreutter DK (1989) A novel antidiabetic agent, CP-68722, increases levels of glucose transport and glucose transporter protein in 3T3-L1 adipocytes. Diabetologia 32: 491A (Abstract)

20. Stevenson RW, Hutson NJ, Krupp MN et al. (1990) Actions of novel antidiabetic agent englitazone in hyperglycemic hyperinsulinemic ob/ob mice. Diabetes 39: 1218-1227

21. Suter SL, Nolan JJ, Wallace P et al. (1992) Metabolic effects of new oral hypoglycemic agent CS-045 in NIDDM subjects. Diabetes Care 15: 193-203

22. Iwamoto Y, Kuzuya T, Matsuda A et al. (1991) Effect of new oral antidiabetic agent CS-045 on glucose tolerance and insulin secretion in patients with NIDDM. Diabetes Care 14: 1083-1086

23. Nolan JJ, Ludvik B, Beerdsen P, Joyce M, Olefsky J (1994) Improvement in glucose tolerance and insulin resistance in obese subjects treated with troglitazone. N Engl J Med 331: 1188-1193

24. National Diabetes Data Group (1979) Classification and diagnosis of diabetes mellitus and other categories of glucose intolerance. Diabetes 28: 1039-1057

25. Amatuzio DS, Shutzman FL, Vanderbilt MJ, Nesbitt S (1953) Interpretation of the rapid IVGTT in normal individuals and in mild diabetes mellitus. $\mathrm{J}$ Clin Invest 32: $438-435$

26. Shimizu S, Tani Y, Yamada H, Tabata M, Murachi T (1980) Enzymatic determination of serum free fatty acid: a colorimetric method. Anal Biochem 107: 193-198

27. Austin M (1989) Plasma trigliceride as a risk factor for coronary heart disease. Am J Epidemiol 129: 249-259 